\title{
Midodrine use in intensive care unit patients recovering from septic shock
}

\author{
Bethannie D Dziuk PharmD, Kenneth Iwuji MD
}

\begin{abstract}
Background: With the rising cost of ICU care and concerns about vasopressor associated complications, clinicians need oral medications that can hasten recovery from septic shock in patients requiring low dose IV vasopressors.

Methods: We retrieved all the available studies published on PubMed and Embase (until September 2018) that reported the use of midodrine in septic shock.

Results: Levine et al reported a decrease in the intravenous vasopressor infusion rate from $-0.62 \pm 1.40 \mathrm{mcg} / \mathrm{min}$ per hour before midodrine to $-2.20 \pm 2.45 \mathrm{mcg} / \mathrm{min}$ per hour after the first four doses of midodrine $(P=0.012)$. Whitson et al reported a two-day decrease in mean ICU length of stay when midodrine was used with IV vasopressors $(P=0.017)$.

Conclusions: Midodrine may have the potential to hasten vasopressor weaning in patients recovering from septic shock, but there are limited published data available to support its use in these patients.
\end{abstract}

Keywords: midodrine, vasopressor, septic shock, alpha (1)-agonist, refractory hypotension, intensive care unit

\section{INTRODUCTION}

The Surviving Sepsis Campaign: International Guidelines for Management of Sepsis and Septic Shock, revised in 2016, provided updated definitions for sepsis and septic shock. Sepsis is currently defined as life-threatening organ dysfunction caused by a dysregulated host response to infection, with septic shock being defined as a subset of sepsis with circulatory and cellular/metabolic dysfunction that is associated with a higher rate of mortality. ${ }^{1-2}$ Clinical criteria were added to the definition of septic shock to aid in the diagnosis and include "hypotension requiring use of vasopressors to maintain a mean arterial pressure of $\geq 65 \mathrm{mmHg}$ and a serum lactate level of

Corresponding author: Kenneth Iwuji Contact Information: Kenneth.Iwuji@ttuhsc.edu. DOI: 10.12746/swrccc.v6i26.509
$>2 \mathrm{mmol} / \mathrm{L}$ persisting after adequate fluid resuscitation." ${ }^{2}$ Furthermore, in 2018, the Surviving Sepsis Campaign released an Hour-1 Bundle, essentially recommending prompt initiation of fluid resuscitation, empiric antibiotic treatment, and application of vasopressors in patients who are hypotensive during or after the initial fluid resuscitation. ${ }^{3}$

In most hospitals, the administration of IV vasopressors occurs in intensive care units (ICUs) where patients are closely monitored for possible side effects and complications from these drugs. Ideally, as the patient's clinical status and hypotension improve, the IV vasopressor is reduced. Occasionally, persistent hypotension in resuscitated patients and dependence on a low dose IV vasopressor lead to delays in discharge from the ICU. The inability to completely wean patients off the IV vasopressors can lead to numerous complications, including an increase in ICU length of stay, peripheral limb ischemia, central line infections and ICU acquired resistant bacterial infections, 
immobilization, ICU delirium, and an overall increase in healthcare costs. Therefore, these complications have led ICU clinicians to look for alternative drugs to facilitate vasopressor weaning, thereby decreasing the ICU length of stay and potential complications.

Some clinicians have used midodrine, an oral, peripherally acting alpha-adrenergic agonist as an off-label weaning drug for refractory hypotension in ICU patients recovering from septic shock. ${ }^{4-5}$ Midodrine received Food and Drug Administration (FDA) approval in 1996 for the treatment of symptomatic orthostatic hypotension. In addition, midodrine has off-labeled indications for the prevention of dialysis-induced hypotension, the treatment of refractory ascites in cirrhotic patients to support diuresis, as combination therapy in hepatorenal syndrome, and treatment of vasovagal syncope..$^{4-5}$

Midodrine, a prodrug, undergoes hepatic metabolism forming desglymidodrine, an active metabolite. Desglymidodrine is an alpha (1)-agonist, which exerts its effects by activating the alpha-adrenergic receptors of the arteriolar and venous vasculature, thus increasing vascular tone and blood pressure (Figure). ${ }^{5}$ In addition to midodrine's ability to increase blood pressure, its pharmacokinetic properties add additional appeal for use as an adjunctive drug. These include a predictable response with rapid absorption after administration with $93 \%$ bioavailability of its active metabolite. ${ }^{4}$ Furthermore, the therapeutic effects of midodrine start within an hour of administration with a duration of 2-3 hours. Peak desglymidodrine levels

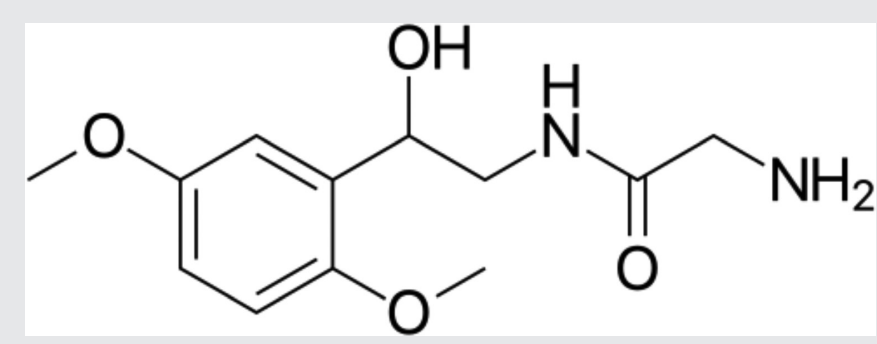

Figure. Chemical structure of midodrine; $\mathrm{C}_{12} \mathrm{H}_{18} \mathrm{~N}_{2} \mathrm{O}_{4}$; molar mass 254.29 grams per mole (Downloaded from Wikimedia- https://commons. wikimedia.org/wiki/File:Midodrine.svg on September 14, 2018). occur about 1-2 hours after administration with a halflife of 3-4 hours. ${ }^{4}$ Midodrine's warning label related to adverse effects includes a marked elevation of supine blood pressure $(>200 \mathrm{mmHg}$ ) and significant bradycardia. Other adverse drug reactions include paresthesia, pruritus, piloerection, chills, abdominal pain, and urinary retention. ${ }^{4}$

Midodrine could hasten vasopressor weaning in ICU patients, but there are limited published data available to support its use in this setting. This review evaluates available data on the use of midodrine as an adjunctive drug for ICU patients recovering from septic shock.

\section{LITERATURE REVIEW}

A prospective, observational study published by in The Journal of Critical Care in 2013 was the first study evaluating the use of midodrine in the ICU setting. ${ }^{6}$ While this study did not explicitly evaluate patients recovering from septic shock, it did demonstrate midodrine's ability to hasten vasopressor weaning and laid the foundation for later and ongoing studies. Briefly, Levine et al evaluated midodrine as adjunct therapy to wean IV vasopressors in patients who otherwise met surgical ICU discharge criteria. Twenty adult patients were included in the study. The most common dose used was midodrine $20 \mathrm{mg}$ PO three times a day. The study reported a decrease in IV vasopressor rate after administration of midodrine from $-0.62 \pm 1.40 \mathrm{mcg} / \mathrm{min}$ per hour before midodrine to $-2.20 \pm 2.45 \mathrm{mcg} / \mathrm{min}$ per hour during the first four doses of midodrine $(P=0.012)$. The median time from the initiation of midodrine to IV vasopressor discontinuation was 17 hours and the median time from initiation of midodrine to ICU discharge was 4 days. Fourteen patients were off IV vasopressors after 24 hours of midodrine treatment. In conclusion, midodrine significantly reduced the duration of IV vasopressor support. ${ }^{6}$

A retrospective, single-center, observational study published by Whitson et al in CHEST in 2016 evaluated the use of oral midodrine to replace IV vasopressors during recovery from septic shock. ${ }^{7}$ This study provided preliminary data to support the use of midodrine as a safe and effective drug to reduce IV vasopressor requirements specifically in ICU patients 
recovering from septic shock. The primary outcome of the study was the duration of IV vasopressor administration and ICU length of stay. The inclusion criteria included patients admitted to the ICU, a diagnosis of septic shock, at least 24 hours of IV vasopressors, and the presence of clinical stability with stable or decreasing doses of IV vasopressors. The exclusion criteria were not specified in the study. A total of 275 were included in the study; 140 patients received only IV vasopressors and 135 received midodrine in addition to the IV vasopressors. Patient baseline characteristics were similar in the two groups. The majority of the sources of sepsis were pulmonary and urinary tract infection in both groups. Results of the primary endpoint demonstrated statistical significance. The IV vasopressor duration was 3.8 days for IV vasopressors only group compared to 2.9 days for the midodrine with IV vasopressors group $(P<0.001)$. Statistically significant differences in the mean ICU length of stay $(9.4 \pm 6.7$ days for the IV vasopressors only group compared to $7.5 \pm 5.9$ days for the midodrine with IV vasopressors group, $\mathrm{P}=0.017$ ) were found. Hospital length of stay, ICU mortality, and hospital mortality did not differ. The authors concluded that the use of midodrine during recovery from septic shock is safe and effective to reduce duration of IV vasopressors and ICU length of stay. ${ }^{7}$

A retrospective, single-center, observational study published in The Journal of Clinical Pharmacy and Therapeutics in 2016 evaluated outcomes in ICU patients who received midodrine. ${ }^{8}$ While this study did not evaluate septic shock explicitly, it did provide pertinent data in this population. The primary outcome of the study was the time to IV vasopressor discontinuation after midodrine initiation. Secondary outcomes of the study included time from IV vasopressor discontinuation to ICU discharge, ICU length of stay, number of ICU readmissions, hospital length of stay, and mean arterial pressure at the time of IV vasopressor initiation and discontinuation. The inclusion criteria included patients 18 years of age or older, admitted to the ICU, receiving one or more IV vasopressors, and an ICD-9 code related to a cardiovascular disease, trauma, or sepsis. Exclusion criteria included patients who died within 24 hours of ICU admission, received IV vasopressors for less than 2 hours, or used midodrine for an indication other than IV vasopressor weaning. The study groups included the intervention group in which patients received at least three doses of midodrine + IV vasopressors and the control group who received IV vasopressors only. A total of 188 patients were included in the study with 94 in the control group and 94 in the midodrine group. Patient baseline characteristics were similar in the two groups except for an APACHE IV score of 59 in the midodrine group compared an APACHE IV score of 83 in the control group. The study reported an overall median time from midodrine initiation to IV vasopressor discontinuation of 1.2 days; the median time in patients with sepsis was 2.2 days. Furthermore, statistical significance was found when comparing the time to ICU discharge after IV vasopressor discontinuation in the midodrine group ( 0.8 days compared to the control group 1.5 days; $P=0.01$ ), hospital length of stay (12 days in the midodrine group compared to 9.5 days in the control group; $P=<0.01$ ), and in-hospital mortality (8 deaths in the midodrine group compared to 21 deaths in the control group; $P=0.01)$. Other important results included significantly more midodrine patients $(79.8 \%$ compared to $46.8 \%$ in the control group) required the addition or the restart of an IV vasopressor one or more times $(P=0.01)$. The authors concluded that midodrine has the potential to be adjunctive treatment in the weaning of IV vasopressors in difficult to wean patients who are otherwise stable. $^{8}$

The results of Levine et al provided preliminary data that support the hypothesis that midodrine may facilitate in weaning ICU patients from an IV vasopressor after the patients' clinical condition stabilize. ${ }^{9}$ This study did not evaluate patients recovering from septic shock specifically, but the results of the study may be extrapolated to this population. Researchers are evaluating midodrine's effects in patients who require low dose IV vasopressors in the Midodrine As Adjunctive Support for the Treatment Of Refractory Hypotension in The Intensive Care Unit: A Multicenter, Randomized, Placebo Controlled Trial (the MIDAS trial); this study is in the recruiting phase. The primary objective of the trial is to determine if the addition of midodrine to standard of care reduces time (hours) to the discontinuation of IV vasopressors. The study will be an international, multicenter, randomized, double blind, placebo-controlled intervention trial. The researchers plan to include 
120 patients who meet the inclusion criteria: adult patients with systolic blood pressure $<90 \mathrm{mmHg}$ who require IV vasopressors for more than 24 hours and are otherwise resuscitated with a stable blood pressure on a single drug. In addition to IV vasopressors, midodrine $20 \mathrm{mg}$ daily or placebo three times day will be evaluated until the primary outcome is achieved or the occurrence of an adverse event. ${ }^{9}$

\section{Conclusion}

With increasing cost and complications associated with ICU care, clinicians need oral medications that can hasten the recovery from septic shock in patients requiring low dose IV vasopressors. In clinical practice, midodrine is being used by many clinicians in patients recovering from septic shock due to its favorable pharmacokinetic profile and direct vasoactive effects. However, there are limited published data to support its use in this setting. Two retrospective studies have reported preliminarily data that support midodrine's potential as an adjunct drug with IV vasopressors. However, results of a randomized, double-blinded, placebo controlled trial are needed to further evaluate its safety and efficacy. While there is currently a randomized-controlled trial underway evaluating midodrine in all ICU patients, there are no ongoing randomized controlled trials to examine efficacy, starting dose, escalation, tapering, and appropriate patient selection for midodrine use during recovery from septic shock.

Article citation: Dziuk BD, Iwuji K. Midodrine use in intensive care unit patients recovering from septic shock. The Southwest Respiratory and Critical Care Chronicles 2018;6(26);26-29

From: Department of Internal Medicine at Texas Tech University Health Sciences Center, Lubbock, Texas (KI); Pharmacy Service at University Medical Center, Lubbock, Texas (BDD)

Submitted: 9/10/2018

Accepted: 9/27/2018

Reviewer: Victor Test MD

Conflicts of interest: none

This work is licensed under a Creative Commons

Attribution-ShareAlike 4.0 International License

\section{REFERENCES}

1. Shankar-Hari M, Phillips GS, Levy ML, et al. Developing a new definition and assessing new clinical criteria for septic shock for the Third International Consensus Definitions for Sepsis and Septic Shock (Sepsis-3). JAMA 2016;315: 775-787.

2. Rhodes A, Evans LE, Alhazzani W, et al. Surviving Sepsis Campaign: international guidelines for management of sepsis and septic shock: 2016. Intensive Care Med. 2017; 43:304-377.

3. Levy MM, Evans LE, Rhodes A, et al. The Surviving Sepsis Campaign Bundle: 2018 Update Crit Care Med 2018 Jun;46(6):997-1000.

4. Midodrine. Lexi-Drugs. Lexicomp. Wolters. Kluwer Health, Inc. Hudson, $\mathrm{OH}$. http://online.lexi.com. Accessed May 16, 2018.

5. ProAmatine [package insert]. Lexington, MA: Shire US Inc; 2017.

6. Levine AR, Meyer MJ, Bittner EA, et al. Oral midodrine treatment accelerates the liberation of intensive care unit patients from intravenous vasopressor infusions. J Crit Care 2013;28(5):756-762.

7. Whitson MR, Mo E, Nabi T, et al. Feasibility, utility, and safety of midodrine during recovery phase from septic shock. Chest 2016;149:1380-1383.

8. Poveromo LB, Michalets EL, Sutherland SE. Midodrine for the weaning of vasopressor infusions. J Clin PharmTherapeuti 2016;41:260-65.

9. Anstey MH, Wibrow B, Thevathasan T, et al. Midodrine as adjunctive support for treatment of refractory hypotension in the intensive care unit: a multicenter randomized, placebo controlled trial (the MIDAS trial). BMC Anesthesiol $2017 \mathrm{Mar}$ 21;17(1):47. 